\title{
Effects of enteral nutrition semi-curing feeding on nutritional diarrhoea improvement in the patients with severe stroke
}

\author{
MAO Zhen-Zhu*, XIONG Fang-Ting*, HU Min, FU Zhuo \\ Department of Neurology, Second Affiliated Hospital of Nanchang University, Nan Chang, China. \\ humin95n@tom.com
}

\begin{abstract}
OBJECTIVE: The present study aims to explore the effects of enteral nutrition (EN) semi-curing feeding on nutritional diarrhoea in the patients with stroke.

METHODS: The patients admitted to the neurological intensive care unit of a tertiary care hospital with stroke- and EN-related diarrhoea between May 2019 and October 2020 were included in the study. The 60 patients, who met the inclusion criteria were divided into the two groups (30 patients each), the experimental group (EN solution + probiotics $+90 \mathrm{ml}$ of pectin) and the control group (EN solution + probiotics), in accordance with the random number table method. The stool number, total stool amount, perineal skin score, and time required to achieve the nutritional target of each patient were recorded at admission and on days 1,3 , and 7 of pectin intervention. The lymphocyte count and the haemoglobin, serum pre-albumin (PA), and total cholesterol (TC) levels were measured in order to assess the patients' nutritional statuses.

RESULTS: The stool number and total stool amount on days 1 and 3 of pectin intervention in the experimental group were better than in the control group; the differences were statistically significant $(p<0.05)$. On day 7 of the intervention, the stool number, total stool amount, and perineal skin score had decreased in the experimental group; the differences were statistically significant, when compared to the control group $(p<0.05)$. Furthermore, the PA levels increased, and TC levels decreased in the experimental group; the differences were statistically significant, when compared to the control group $(p<0.05)$. On day 3 of the intervention, the PA levels in the experimental group were increased compared to the control group; the difference was statistically significant $(p<0.05)$.

CONCLUSION: EN semi-curing feeding could improve EN-related diarrhoea and nutritional status levels in patients with stroke (Tab. 3, Ref. 9). Text in PDF www.elis.sk KEY WORDS: severe stroke, pectin, enteral nutrition, diarrhoea, semi-curing.
\end{abstract}

\section{Introduction}

Enteral nutrition (EN) can effectively maintain the normal intestinal mucosal cell structure; promote intestinal motility, secretion, and digestion; regulate systemic immune function; and improve patient nutritional status. Furthermore, it is considered the ideal nutritional support method (1). Ortiz-Reyes et al (2) found that the use of EN in critically ill patients significantly reduced infection incidence and decreased patient morbidity and mortality. The study conducted by Hua et al (3) indicated that an early EN application in patients with stroke could help to improve their neurological function, disease recovery, and prognosis. EN support

Department of Neurology, Second Affiliated Hospital of Nanchang University, Nan Chang, China

Address for correspondence: Min Hu, Department of Neurology, The Second Affiliated Hospital of Nanchang University, No. 1, Minde Road, Nanchang 330006, China.

Phone: +86.13177864961 , Fax: +86.1083161294

*These authors contributed equally to this study

Funding: Science and Technology Program of Jiangxi Provincial Health Commission (No. 20204309). is not only a nutrient supplement method, but also an important therapeutic measure. The EN procedure is often accompanied by a nutritional intolerance in patients. The most common type of EN-induced nutritional intolerance is diarrhoea, which can lead to water and electrolyte imbalance, intestinal flora translocation, and an increased infection fatality incidence. Kozeniecki et al (4) suggested that the EN diarrhoea incidence in critically ill patients can reach up to $66 \%$. The patients with severe stroke are in a state of stress due to serious trauma, infection, dysphagia, etc. They are prone to develop malnutrition and require EN. Therefore, it is crucial that effective prevention and intervention measures are taken to improve EN-related diarrhoea in critically ill patients. Clinical methods, such as using special EN pumps and thermostatic heaters, changing the type of nutrition solution, and adding probiotics to minimize the gastrointestinal tract burden, can reduce nutritional diarrhoea to a certain extent and facilitate nutrient absorption; however, the effects of these methods are not statistically significant $(5,6)$. In recent years, related studies $(7)$ have shown that the combined application of pectin and EN preparations forms a semi-curing state that is more effective in improving the prevention of diarrhoea, reducing intestinal bacterial translocation, and significantly improving intestinal function in patients. Therefore, 
the present study observed the effects of pectin on diarrhoea by applying pectin to patients with stroke and EN-related diarrhoea, providing more effective clinical treatment ideas for EN-induced diarrhoea in the neurological intensive care unit (NICU).

\section{Methods}

\section{Patients}

The patients admitted to the NICU of the tertiary care hospital in Nanchang with stroke- and EN-related diarrhoea between May 2019 and October 2020 were selected as subjects for the present study. According to the inclusion and exclusion criteria, a total of 60 patients ( 36 males and 24 females) were enrolled. The patients were aged $40-70$, with the average age of $58.6 \pm 9.5$ years. There were 37 cases with cerebral infarction and 23 cases with cerebral haemorrhage among the patients. There were no significant differences in age, gender, and disease severity between the two groups $(\mathrm{p}>0.05)$. The patient diagnoses were made in accordance with the diagnostic criteria for severe stroke established in the 2015 China Consensus on the Management of Severe Cerebrovascular Diseases.

Inclusion criteria: (1) patients with a consciousness disorder and a Glasgow coma score of $\leq 8$; (2) patients with an acute physiology and chronic health evaluation II score of $\geq 15$ and an estimated NICU stay length of over seven days; and (3) patients, who received early EN support within $24-48 \mathrm{~h}$ after admission to the NICU and experienced nutritional diarrhoea. Exclusion criteria: (1) patients with severe diseases, such as: renal failure, myocardial infarction, and multiple organ dysfunction syndrome; (2) patients, who had infectious diarrhoea before an admission to the NICU; (3) patients with a history of gastrointestinal surgery or gastrointestinal diseases; (4) patients with severe immunodeficiency or malignant tumours; and (5) patients with incontinent dermatitis.

\section{Preparation}

The dietary fibres used were: (1) Wei shuneng Pectin: Qixinliang (Hangzhou) Biotechnology Co., Ltd., production license number SC10633012712541, specifications 90 g/bag containing $2.0 \mathrm{~g}$ fibre per $100 \mathrm{~g}$; (2) Jiaxian Nutritional Liquid Food (Li Shikang): Xi'an Libang Clinical Nutrition Co., Ltd., production license number SC130610111200721, specifications $500 \mathrm{ml} /$ bottle, containing $3.6 \mathrm{~g}$ of protein, $1.2 \mathrm{~g}$ of dietary fibre, and 411 KJ of calories per $100 \mathrm{ml}$; and (3) Licheng Probiotic Granules: Shanghai Licheng Nutrition Product Technology Co., Ltd., specifications $2 \mathrm{~g} / \mathrm{b}$ ar and a live bacteria content of $\geq 1 \times 1010 \mathrm{CFU} / \mathrm{bar}$ at production.

\section{Specific implementation method}

The 60 patients, who met the inclusion criteria were divided into two groups (30 patients each), the experimental group (EN solution + probiotics $+90 \mathrm{ml}$ of pectin) and the control group (EN solution + probiotics), in accordance with the random number table method. All the enrolled patients received routine symptomatic treatment, which mainly included dehydration to lower an intra- cranial pressure, improve cerebral circulation, and nourish nerves. The EN support treatment was administered via a nasal feeding tube and began in all the patients within $24-48 \mathrm{~h}$ after admission to the NICU. The patient daily caloric supply standard followed the 2006 Guiding Opinions on Nutrition Support for Critical Patients by the Critical Care Medicine Branch of the Chinese Medical Association, with a target daily caloric intake of $30 \mathrm{kcal} / \mathrm{kg} \cdot \mathrm{d}$. The EN solution was continuously infused through a constant-temperature heater. On day 1 of EN administration, $500 \mathrm{ml}$ of pectin was infused and pumped at a rate of $40 \mathrm{ml} / \mathrm{h}$, and the stomach contents were withdrawn q6 h. In the case of no adverse reactions, the dosage should be increased to $1000 \mathrm{ml} /$ day and pumped at $80 \mathrm{ml} / \mathrm{h}$. The administration speed and dosage should be adjusted reasonably according to patient intestinal tolerance, and in the case of a stomach content withdrawal of $>150 \mathrm{ml}$, the infusion should be suspended and reassessed after $2 \mathrm{~h}$. In the present study, probiotics were added to the daily EN solution infusion. The probiotics were dissolved in $20 \mathrm{ml}$ of warm water and injected through a nasal feeding tube three times a day. The detailed nutritional support methods in the two groups were as follows: in the control group, nutritional support was conducted concerning the above-mentioned standards, and in the experimental group, pectin was added to the control group treatments with the occurrence of diarrhoea, and $90 \mathrm{ml}$ (1 bag) of pectin was injected through the nasal tube three times a day before the daily EN infusion. The detailed steps were as follows: (1) the nasal tube was flushed with $20 \mathrm{ml}$ of boiled warm water; (2) $90 \mathrm{ml}$ (1 bag) of Wei shuneng Pectin was drawn and infused through the end of the nasal tube; (3) the nasal tube was flushed again with $20 \mathrm{ml}$ of boiled warm water; and (4) the EN solution was infused.

\section{Diarrhoea}

The stool number and total stool amount in the patients were observed before EN support and on days 1, 3, and 7 of pectin intervention. Hart's diarrhoea scoring method, developed by Hart $\mathrm{G} \mathrm{K}$ in 1998, was adopted for this procedure. The method focuses on adding up the estimated stool volume for each 24 -h period and the stool property scores in order to obtain the total daily score. A total score of $>12$ indicates diarrhoea.

\section{Perineal skin}

The perineal assessment tool was used to determine the perineal skin condition in the two groups. The tool contains four items: (1) stimulant type; (2) stimulation duration; (3) perineal skin condition; and (4) influencing factors. The results are based on a 3-point scoring method, with each item divided into 3 levels (1, 2, and 3 points, each) and a total score of $4-12$. The higher the score, the worse the skin condition. The range of the low-risk score is $4-6$, and the range of the high-risk score is $7-12$.

\section{Nutritional status}

Blood samples were drawn to determine the lymphocyte count and the serum haemoglobin $(\mathrm{Hb})$, serum pre-albumin (PA), and total cholesterol (TC) levels before EN support administration and on days 1,3 , and 7 of pectin intervention. 


\section{4-217}

\section{Statistical analysis}

The Statistical Package for Social Sciences 23.0 software was adopted for data analysis. The measurement data conforming to a normal distribution were described using the mean \pm standard deviation $\left(\bar{\chi}^{ \pm s}\right)$, and comparisons between the two groups were made using a one-way analysis of variance. The countable data were statistically described with percentages, and comparisons between the two groups were performed using the chi-square test. The $\mathrm{p}$-value $<0.05$ was considered statistically significant.

\section{Results}

Comparison of diarrhoea and perineal skin scores between the patient groups

There were no statistically significant differences in the stool number, total stool amount, and perineal skin scores between the two groups before the intervention $(p>0.05)$. On days 1 and 3 of pectin intervention, the stool number and total stool amount in the experimental group decreased more than in the control group; the differences were statistically significant $(\mathrm{p}<0.05)$. The stool number, total stool amount, and perineal skin score in the experimental group showed a significant decrease on day 7 of pectin intervention, with statistically significant differences between the experimental group and the control group $(\mathrm{p}<0.05)$. The details are illustrated in Table 1.

\section{Comparison of the patient group nutritional statuses}

There were no statistical differences in the lymphocyte count and the serum $\mathrm{Hb}, \mathrm{PA}$, and TC levels between the two groups before pectin intervention and on day 1 of the intervention $(p>$ 0.05 ). On day 3 of the intervention, the serum PA levels in the experimental group were increased compared to the control group; the difference was statistically significant $(\mathrm{p}<0.05)$. On day 7 of the intervention, the serum PA levels increased, and the TC levels decreased in the experimental group; the differences were statistically significant when compared to the control group $(\mathrm{p}<0.05)$ (Tab. 2)

Tab. 1. Comparison of diarrhoea and perineal skin scores between patient groups $(\bar{\chi} \pm s)$.

\begin{tabular}{lccccc}
\hline & $\begin{array}{c}\text { Pectin } \\
\text { intervention } \\
\text { days }\end{array}$ & $\begin{array}{c}\text { Experimental } \\
\text { group }\end{array}$ & $\begin{array}{c}\text { Control } \\
\text { group }\end{array}$ & $\mathrm{Z}$ & $\mathrm{P}$ \\
Stool & 0 & $4.23 \pm 0.37$ & $4.13 \pm 0.29$ & 0.88 & 0.831 \\
number & 1 & $2.33 \pm 0.41$ & $3.93 \pm 0.33$ & 9.04 & 0.032 \\
& 3 & $1.49 \pm 0.18$ & $3.82 \pm 0.34$ & 10.69 & 0.011 \\
& 7 & $0.87 \pm 0.16$ & $3.70 \pm 0.12$ & 15.83 & 0.001 \\
\hline \multirow{2}{*}{ Total stool } & 1 & $259.67 \pm 29.77$ & $363.00 \pm 23.36$ & 1.26 & 0.041 \\
amount & 3 & $153.68 \pm 19.30$ & $253.74 \pm 25.12$ & 15.78 & 0.001 \\
& 7 & $63.67 \pm 16.93$ & $193.00 \pm 18.34$ & 12.81 & 0.004 \\
\hline Perineal & 0 & $8.03 \pm 0.41$ & $8.07 \pm 0.52$ & 1.13 & 0.770 \\
skin & 1 & $8.01 \pm 0.25$ & $8.08 \pm 0.54$ & 1.19 & 0.813 \\
scores & 3 & $6.87 \pm 0.31$ & $7.57 \pm 0.42$ & 2.59 & 0.769 \\
& 7 & $4.70 \pm 0.29$ & $7.27 \pm 0.54$ & 43.48 & 0.001 \\
\hline
\end{tabular}

Tab. 2. Comparison of patient group nutritional statuses $(\bar{\chi} \pm s)$.

\begin{tabular}{|c|c|c|c|c|c|}
\hline & $\begin{array}{c}\text { Pectin } \\
\text { intervention } \\
\text { days }\end{array}$ & $\begin{array}{l}\text { Experimental } \\
\text { group }\end{array}$ & $\begin{array}{l}\text { Control } \\
\text { group }\end{array}$ & $\mathrm{F}$ & $\mathrm{P}$ \\
\hline \multirow{4}{*}{$\begin{array}{l}\text { Serum } \\
\text { haemoglobin } \\
(\mathrm{g} / \mathrm{l})\end{array}$} & 0 & $115.20 \pm 21.02$ & $116.87 \pm 23.62$ & 0.72 & 0.892 \\
\hline & 1 & $115.83 \pm 20.52$ & $116.07 \pm 21.96$ & 0.67 & 0.932 \\
\hline & 3 & $117.18 \pm 22.47$ & $115.60 \pm 24.15$ & 1.52 & 0.231 \\
\hline & 7 & $119.40 \pm 26.19$ & $113.20 \pm 25.21$ & 2.58 & 0.092 \\
\hline \multirow{4}{*}{$\begin{array}{l}\text { Serum } \\
\text { pre-albumin } \\
(\mathrm{mg} / \mathrm{l})\end{array}$} & 0 & $142.85 \pm 58.32$ & $151.74 \pm 54.19$ & 0.52 & 0.792 \\
\hline & 1 & $141.95 \pm 58.74$ & $150.39 \pm 53.46$ & 0.43 & 0.732 \\
\hline & 3 & $158.32 \pm 63.58$ & $138.94 \pm 58.82$ & 3.63 & 0.015 \\
\hline & 7 & $178.35 \pm 62.20$ & $108.40 \pm 59.21$ & 4.16 & 0.008 \\
\hline \multirow{4}{*}{$\begin{array}{l}\text { Total } \\
\text { cholesterol } \\
(\mathrm{mmol} / \mathrm{L})\end{array}$} & 0 & $3.56 \pm 0.53$ & $3.57 \pm 0.59$ & 2.14 & 0.195 \\
\hline & 1 & $3.54 \pm 0.77$ & $3.64 \pm 0.76$ & 2.68 & 0.120 \\
\hline & 3 & $3.50 \pm 0.92$ & $3.89 \pm 0.83$ & 3.58 & 0.058 \\
\hline & 7 & $2.84 \pm 0.76$ & $3.93 \pm 0.93$ & 4.16 & 0.017 \\
\hline \multirow{4}{*}{$\begin{array}{l}\text { Lymphocyte } \\
\text { count } \\
\left(\times 10^{9} \mathrm{~L}\right)\end{array}$} & 0 & $0.96 \pm 0.29$ & $0.97 \pm 0.33$ & 0.59 & 0.682 \\
\hline & 1 & $1.02 \pm 0.33$ & $1.01 \pm 0.34$ & 1.57 & 0.573 \\
\hline & 3 & $1.29 \pm 0.42$ & $1.21 \pm 0.31$ & 1.53 & 0.492 \\
\hline & 7 & $1.45 \pm 0.36$ & $1.31 \pm 0.33$ & 2.09 & 0.098 \\
\hline
\end{tabular}

Tab. 3. Comparison of the time required to achieve the target EN amount between patient groups $\left(\bar{\chi}^{ \pm s}\right)$.

\begin{tabular}{lcccc}
\hline & $\begin{array}{c}\text { Experimental } \\
\text { group }\end{array}$ & $\begin{array}{c}\text { Control } \\
\text { group }\end{array}$ & $\mathrm{Z}$ & $\mathrm{P}$ \\
\hline Minimum time to reach target (d) & 3.0 & 5.0 & - & - \\
Time to reach the target (d) & $4.22 \pm 0.57$ & $6.19 \pm 0.82$ & 33.59 & 0.004 \\
\hline
\end{tabular}

\section{Comparison of the time required to achieve the target EN amount} between patient groups

Patients in the experimental group took less time to reach the target EN amount than patients in the control group; the difference was statistically significant $(\mathrm{p}<0.05)$ (Tab. 3 ).

\section{Discussion}

EN-related diarrhoea is defined as different degrees of bloating and diarrhoea in patients after two days of EN, with a symptom relief after the adjustment of the concentration, temperature, infusion rate, or infusion volume of the nutrition solution, along with diarrhoea exclusion caused by other factors. The occurrence of diarrhoea leads to water-electrolyte disorders, incontinent dermatitis, and increased morbidity and mortality, all of which seriously affect patient recovery and prognosis.

The EN semi-curing feeding was effective in stool number and total stool amount reduction as well as perineal skin score improvement in patients with stroke

In recent years, several studies have confirmed that adding dietary fibre to EN application in critically ill patients can significantly improve their intestinal function, protect the intestinal mucosal barrier, treat constipation, improve diarrhoea, reduce intestinal bacterial translocation, and control blood glucose. Among these, the effects on diarrhoea improvement are particularly evident. The results of the present study showed that the stool number and total stool amount on days 1 and 3 of pectin intervention in the experimental group decreased more significantly than in the 
control group, and the differences were statistically significant $(\mathrm{p}<0.05)$. The stool number, total stool amount, and perineal skin score in the experimental group decreased significantly on day 7 of the intervention, with statistically significant differences between the experimental group and the control group $(\mathrm{p}<0.05)$.

EN semi-curing feeding could lead to pre-albumin elevation and a faster TC decrease in the patients with stroke

Neurocritically ill patients usually have a high catabolic metabolism and may also have an inadequate nutritional intake due to impaired consciousness, autonomic dysfunction, or malabsorption. With the emergence of theories on ectopic intestinal flora, intestinal mucosal barrier protection, and intestinal immune function, the importance of EN for the patients with stroke has been receiving an increasing attention $(8,9)$. Pectin can be used as an adherent and protective carrier for intestinal flora, which cannot be digested by the human gastrointestinal tract and can stimulate gastrointestinal peristalsis. Pectin can further protect the intestinal mucosal barrier, reduce ectopic intestinal flora, improve the intestinal immune function and the level of albumin as well as the nutritional status, and accelerate the recovery process in the patients with stroke (1).

EN semi-curing feeding could help patients with stroke to reach the target EN amount as soon as possible

In the present study, the above-mentioned target nutritional amount was the target EN caloric amount calculated according to the patient's body mass. Parenteral nutrition calories, such as fatty milk, glucose, and amino acids, were not included. The results suggest that EN combined with pectin not only improved the intestinal condition in the patients with stroke but also increased the duration of nutrient retention in the patients' intestines. Hence, the experimental group reached the target nutrition amount sooner than the control group, and its patient nutritional status levels were improved.

\section{Conclusion}

In conclusion, an early addition of pectin to EN therapy in critically ill patients could effectively improve nutritional diarrhoea, help to achieve the target feeding amount as soon as possible, and reduce complications caused by diarrhoea, making it worthy of clinical promotion and application.

\section{References}

1. Wei J, Jiang R, Li L, Kang D, Gao G, You C, Zhang J, Gao L, Huang Q, Luo D, Zhao G, Zhang H, Wang S, Wang R. Stress-related upper gastrointestinal bleeding in adult neurocritical care patients: a Chinese multicenter, retrospective study. Curr Med Res Opin 2019; 35: 181-187.

2. Ortiz-Reyes LA, Chang Y, Quraishi SA, Yu L, Kaafarani H, de Moya M, King DR, Fagenholz P, Velmahos G, Yeh DD. Early Enteral Nutrition Adequacy Mitigates the Neutrophil-Lymphocyte Ratio Improving Clinical Outcomes in Critically Ill Surgical Patients. Nutr Clin Pract 2019; 34: 148-155.

3. Hua YL. (Effect of early enteral nutrition on prognosis of patients with large area cerebral infarction). Chin J Pract Nerv Dis 2019; 22: 2264-2269.

4. Kozeniecki M, Fritzshall R. Enteral Nutrition for Adults in the Hospital Setting. Nutr Clin Pract 2015; 30: 634-651.

5. Lee HJ, Choi JK, Ryu HS, Choi CH, Kang EH, Park KS, Min YW, Hong KS. Therapeutic Modulation of Gut Microbiota in Functional Bowel Disorders. J Neurogastroenterol Motil 2017; 23: 9-19.

6. Chang SJ, Huang HH. Diarrhea in enterally fed patients: blame the diet? Curr Opin Clin Nutr Metab Care. 2013; 16: 588-94.

7. Nakamura K, Inokuchi R, Fukushima K, Naraba H, Takahashi Y, Sonoo T, Hashimoto H, Doi K, Morimura N. Pectin-containing liquid enteral nutrition for critical care: a historical control and propensity score matched study. Asia Pac J Clin Nutr 2019; 28: 57-63.

8. Vieira LV, Pedrosa LAC, Souza VS, Paula CA, Rocha R. Incidence of diarrhea and associated risk factors in patients with traumatic brain injury and enteral nutrition. Metab Brain Dis 2018; 33: 1755-1760.

9. Scerrati A, De Rosa S, Mongardi L, Cavallo MA, Trapella G, De Bonis P. Standard of care, controversies, and innovations in the medical treatment of severe traumatic brain injury. J Neurosurg Sci 2018; 62: 574-583.

Received September 9, 2021. Accepted November 23, 2021. 